\title{
Shape Dependent Macromolecular Crowding on the Thermodynamics and Microsecond Conformational Dynamics of Protein Unfolding Revealed at the Single Molecular Level
}

\author{
Nilimesh Das and Pratik Sen* \\ Department of Chemistry, Indian Institute of Technology Kanpur, Kanpur - 208 016, UP, India \\ * Corresponding author, E-mail: psen@iitk.ac.in, Fax: +91-512-259-6808
}

\section{Content:}

Section S1: Synthesis of $p$-nitrophenyl coumarin ester (NPCE)

Section S2: Protein labelling

Section S3: FCS instrumentation

Section S4: FCS data fitting procedure

Section S5: Discussion on the covalent tagging of NPCE to Tyr-411 resideu

Table S1: Secondary structural parameters of untagged and NPCE tagged HSA

Table S2: $\%$ of $\alpha$-helicity of HSA at various temperatures in the absence and presence of crowders.

Table S3: Emission maxima of NPCE labelled to domain-III of HSA at various temperatures in the absence and presence of crowders

Table S4: Hydrodynamic radius $\left(r_{H}\right)$ and conformational fluctuation time $\left(\tau_{R}\right)$ of HSA at various temparatures in the absence and presence of crowders under investigation

Figure S1: Circular dichroism spectra of untagged (blue) and NPCE tagged (red) HSA.

Figure S2: Autocorrelation curve of free NPCE (cyan) and NPCE tagged to HSA (red) in buffer

Figure S3: Variation of Hydrodynamic radius of NPCE tagged HSA with increasing the temperature from $15{ }^{\circ} \mathrm{C}$ to $70{ }^{\circ} \mathrm{C}$ (unfolding) and with decreasing temperature from $70{ }^{\circ} \mathrm{C}$ to $15{ }^{\circ} \mathrm{C}$ (folding)

Figure S4: Variation of Hydrodynamic radius of NPCE tagged HSA with increasing the temperature from $15{ }^{\circ} \mathrm{C}$ to $80{ }^{\circ} \mathrm{C}$ (unfolding) and with decreasing temperature from $80{ }^{\circ} \mathrm{C}$ to $15{ }^{\circ} \mathrm{C}$ (folding) 


\section{Section S1: Synthesis of $p$-nitrophenyl coumarin ester (NPCE)}

We took the equimolar amount of coumarin-343, para-nitrophenol and 4-dimethylamino pyridine (DMAP) in dichloromethane and stirred them in an ice bath for 10 mins. Equimolar $\mathrm{N}, \mathrm{N}$-dicyclohexylcarbodiimide (DCC) was added under nitrogen atmosphere while stirring. The solution was stirred at $0^{\circ} \mathrm{C}$ in an ice bath for further 20 mins and then at $20^{\circ} \mathrm{C}$ for 24 hours. We then washed the organic layer with $\sim 35 \mathrm{ml} 1.2 \mathrm{M} \mathrm{HCl}$ and then with $35 \mathrm{ml}$ of saturated $\mathrm{NaHCO}_{3}$ solution. It was then dried over $\mathrm{MgSO} 4$. We then suspended the residue in methanol and washed the precipitate with $100 \mathrm{~mL}$ of methanol to remove the unreacted coumarin-343. The precipitate was collected in dichloromethane, dried under vacuum and characterized by $1 \mathrm{H}-\mathrm{NMR}$, IR and mass spectrometric studies.

\section{Section S2: Protein Labeling}

We dissolved $50 \mathrm{mg}$ of HSA, and $0.4 \mathrm{mg}$ of NPCE in $9 \mathrm{~mL}$ of $50 \mathrm{mM}$ phosphate buffer $(\mathrm{pH}$ $=8.0$ ) and $)$ and in a minimum volume of DMSO respectively. Then these two solutions are very slowly mixed while stirring. The reaction mixture was then stirred at the room temperature for $30 \mathrm{hrs}$. Then dialysis was done to remove any untagged dye against $500 \mathrm{~mL}$ of 1:15 (v/v) DMSO and buffer (pH 7.4, $50 \mathrm{mM}$ phosphate buffer) solution at $5{ }^{\circ} \mathrm{C}$ for 4 days and then against only phosphate buffer for another 4 days. We changed the dialysis medium every 12 hours and recorded the fluorescence of the dialysis medium to check whether the dialysis is complete or not. Dialysis is stopped when we do not get any appreciable fluorescence from the dialyzed solution.

\section{Section S3: FCS Instrumentation}

We use an inverted microscope (IX-71, Olympus, Japan) for this setup. The excitation source is $405 \mathrm{~nm} \mathrm{CW}$ laser (MGL-III-532-5mW, CNI, China). The light passes through a beam expander (to overfill the back of the objective), several mirrors to a 60Xwater immersion objective with numerical aperture 1.2 and is focussed into the sample at a distance of $40 \mu \mathrm{m}$ from the surface of the cover-slip attache to the bottom of a home built temperature controlled sample holder. The temperature of the cellis controlled using a temperature controlled water circulation system (LLCB-202, Labocon, UK). The same objective collects the emitted photons and focuses on a multimode fiber patch cord of $25 \mu \mathrm{m}$ diameter after passing through a dichroic mirror (ZT405rdc, Omega Optical Inc., USA) and an emission filter (FSQ-GG455, USA). The fluorescence signal isthen directed toa photon counting 
module (SPCM-AQRH-13-FC, Excelitas, Canada) and then to the hardware correlator (FLEX990EM-12E, Correlator.com, USA) to generate the fluorescence intensity autocorrelation function.

\section{Section S4: FCS data fitting Procedure}

Autocorrelation function $G(\tau)$ that arises because of the temporal fluctuation of the fluorescence intensiy can be described as,

$G(\tau)=\frac{\langle\delta F(t) \delta F(t+\tau)\rangle}{\langle F(t)\rangle^{2}}$

The autocorrelation is the self-similarity of fluorescence intensity at different times. $\langle F(t)$ is the average fluorescence intensity, and $\delta F(\tau)$ and $\delta F(t+\tau)$ are the quantity of fluctuation in intensity around the mean value at time $t$ and $(t+\tau)$;

$\delta F(t+\tau)=F(t+\tau)-\langle F(t)\rangle$

For a single component system, the diffusion time $\left(\tau_{D}\right)$ can be obtained by fitting the autocorrelation function $G(\tau)$ using the following equation

$G(\tau)=\frac{1}{N}\left(1+\frac{\tau}{\tau_{D}}\right)^{-1}\left(1+\frac{\tau}{\omega^{2} \tau_{D}}\right)^{-1 / 2}$

Where, $N$ is the number of particles in the observation volume and $\omega=l / r$ is the longitudinal to transverse radius ratio of the 3D Gaussian volume. If the diffusing species undergoes any other process having amplitude $A$ and timescale $\tau_{\mathrm{R}}$ that gives rise to additional fluorescence fluctuation, the modified correlation function can be writtenas:

$G(\tau)=\frac{1}{N}\left(1+\frac{\tau}{\tau_{D}}\right)^{-1}\left(1+\frac{\tau}{\omega^{2} \tau_{D}}\right)^{-1 / 2}\left(1+A \cdot \exp \left(-\frac{\tau}{\tau_{R}}\right)\right)$

We estimated the detection volume to be $0.7 \mathrm{fL}$ using the equation...

$V_{e f f}=\pi^{\frac{3}{2}} r^{3} \omega$

\section{Section S5: Discussion on the covalent tagging of NPCE to Tyr-411 residue}

There are altogether 19 tyrosine resideus are present in HSA. However, Tyr-411 is the most accessible one at $\mathrm{pH} 8$ for esterification reaction owing to its considerably low $\mathrm{pK}_{\mathrm{a}}$ value. ${ }^{\mathrm{R} 1}$ The ratio of the protein and the dye (NPCE) is taken as 1:0.87 to avoid tagging of NPCE at multiple sites. We have also proved this site specific tagging by various spectroscopic technique. The blue shift of NPCE of $7 \mathrm{~nm}$ and $9 \mathrm{~nm}$ in the absorption and emission spectra respectively as shown below. This proves that it enters more hydrophobic protein matrix as compared to water. Now, we have confirmed that NPCE selectively binds to the Tyr-411 
residue, in two ways. We have calculated the ratio of HSA: NPCE in the tagged protein to be 0.8. At reaction condition $(\mathrm{pH}=8)$ Tyr-411 is way more reactive than the other tyrosine residues [R1]. Combining these two facts, we can confirm the selective tagging. The fact that the diffusion time of NPCE tagged to HSA $(132 \mu \mathrm{s})$ is much higher as compared to free NPCE in the buffer $(20 \mu \mathrm{s})$ also proves the tagging. Further, NPCE labeled to HSA has been satisfactorily fitted with equation (4) suggesting only one conformational fluctuation time. Had the tagging occur in multiple sites, we should not get such proper fitting by the above equation. This also proves that NPCE is covalently attached to only one place of HSA, and being the most reactive site, Tyr-411 is the site.

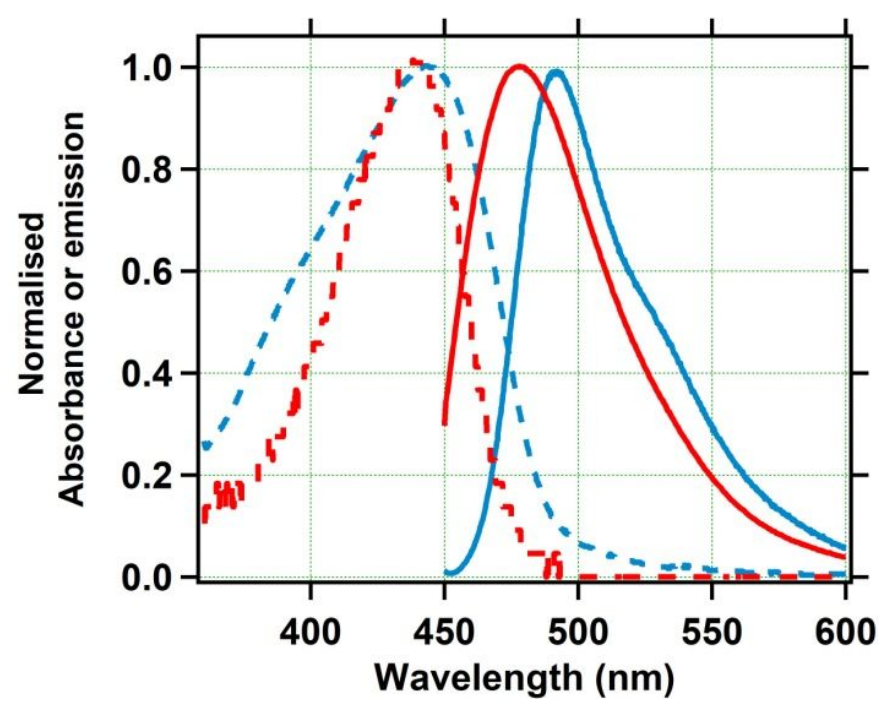

Normalised absorption (dotted line) and emission spectra (solid line) of free NPCE (cyan) and NPCE tagged to HSA (red line).

Table S1: Secondary structural parameters of untagged and NPCE tagged HSA.

\begin{tabular}{|c|c|c|c|c|}
\hline \multirow{2}{*}{} & \multicolumn{4}{|c|}{$\%$-of secondary Structural contents } \\
\cline { 2 - 5 } & $\boldsymbol{\alpha}$-helicity & $\boldsymbol{\beta}$-sheet & $\boldsymbol{\beta}$-turn & Random coil \\
\hline Untagged HSA & 68.0 & 5.8 & 12.0 & 14.2 \\
\hline NPCE tagged HSA & 65.6 & 6.0 & 12.4 & 16.0 \\
\hline
\end{tabular}

Table S2: $\%$ of $\alpha$-helicity of HSA at various temperatures in the absence and presence of crowders under investigation.

\begin{tabular}{|l|l|l|l|l|}
\hline \multirow{2}{*}{ Temperature } & \multicolumn{4}{|c|}{$\%$ of $\boldsymbol{\alpha}$-helicity of HSA } \\
\cline { 2 - 5 } & No Crowder & Dextran-40 & Ficoll-70 & PEG-35 \\
\hline $\mathbf{1 5}^{\mathbf{}} \mathbf{C}$ & 66.4 & 70.0 & 71.6 & 77.0 \\
\hline
\end{tabular}




\begin{tabular}{|l|l|l|l|l|}
\hline $\mathbf{2 0}{ }^{\circ} \mathbf{C}$ & 66.0 & 70.6 & 71.4 & 77.2 \\
\hline $\mathbf{2 5}{ }^{\circ} \mathbf{C}$ & 65.4 & 70.5 & 70.2 & 76.8 \\
\hline $\mathbf{3 0}^{\circ} \mathbf{C}$ & 65.1 & 71.0 & 69.5 & 77.2 \\
\hline $\mathbf{3 5}^{\circ} \mathbf{C}$ & 64.2 & 70.6 & 68.1 & 75.9 \\
\hline $\mathbf{4 0}^{\circ} \mathbf{C}$ & 63.5 & 68.8 & 68 & 74.2 \\
\hline $\mathbf{4 5}^{\circ} \mathbf{C}$ & 62.3 & 68.2 & 67.4 & 73.5 \\
\hline $\mathbf{5 0}^{\circ} \mathbf{C}$ & 60.0 & 67.2 & 66.7 & 71.4 \\
\hline $\mathbf{5 5}^{\circ} \mathbf{C}$ & 53.3 & 65.0 & 64 & 67.3 \\
\hline $\mathbf{6 0}^{\circ} \mathbf{C}$ & 50.5 & 56.4 & 59.4 & 63.9 \\
\hline $\mathbf{6 5}^{\circ} \mathbf{C}$ & 46.7 & 48.2 & 53.5 & 54.1 \\
\hline $\mathbf{7 0}^{\circ} \mathbf{C}$ & 39.2 & 41.9 & 49.7 & 43.0 \\
\hline
\end{tabular}

Table S3: Emission maxima of NPCE labelled to domain-III of HSA at various temperatures in the absence and presence of crowders under investigation.

\begin{tabular}{|l|l|l|l|l|}
\hline \multirow{2}{*}{ Temperature } & \multicolumn{4}{|c|}{ Emission Maxima (nm) } \\
\cline { 2 - 5 } & No Crowder & Dextran-40 & Ficoll-70 & PEG-35 \\
\hline $\mathbf{1 5}{ }^{\mathbf{C}} \mathbf{C}$ & 477.2 & 477 & 477 & 477.6 \\
\hline $\mathbf{2 0}{ }^{\mathbf{C}} \mathbf{C}$ & 477.2 & 477 & 477 & 477.5 \\
\hline $\mathbf{2 5}{ }^{\mathbf{C}}$ & 477.2 & 477 & 477 & 477.7 \\
\hline $\mathbf{3 0}^{\mathbf{C}} \mathbf{C}$ & 477.2 & 477 & 477.1 & 477.8 \\
\hline $\mathbf{3 5}^{\mathbf{C}} \mathbf{C}$ & 477.2 & 477 & 477.2 & 477.9 \\
\hline $\mathbf{4 0}^{\mathbf{C}} \mathbf{C}$ & 477.2 & 477 & 477.2 & 478 \\
\hline $\mathbf{4 5}^{\mathbf{0}} \mathbf{C}$ & 477.7 & 477.5 & 477.2 & 478.2 \\
\hline $\mathbf{5 0}^{\mathbf{0}} \mathbf{C}$ & 478 & 477.5 & 477.5 & 478.3 \\
\hline $\mathbf{5 5}^{\mathbf{C}} \mathbf{C}$ & 478.2 & 478 & 477.8 & 478.8 \\
\hline $\mathbf{6 0}^{\mathbf{0}} \mathbf{C}$ & 479.2 & 478.5 & 478.6 & 480 \\
\hline $\mathbf{6 5}^{\mathbf{0}} \mathbf{C}$ & 480.2 & 479.5 & 479.5 & 481.2 \\
\hline $\mathbf{7 0}^{\mathbf{0}} \mathbf{C}$ & 481.2 & 481 & 480.6 & 482.9 \\
\hline
\end{tabular}

Table S4: Hydrodynamic radius $\left(r_{H}\right)$ and conformational fluctuation time $\left(\tau_{R}\right)$ of HSA at various temparatures in the absence and presence of crowders under investigation.

\begin{tabular}{|l|l|l|l|l|l|l|l|l|}
\hline \multirow{2}{*}{$\begin{array}{c}\text { Temperatur } \\
\text { e }\end{array}$} & \multicolumn{2}{|c|}{ No Crowder } & \multicolumn{2}{c|}{ Dextran-40 } & \multicolumn{2}{c|}{ Ficoll-70 } & \multicolumn{2}{c|}{ PEG-35 } \\
\cline { 2 - 9 } & $\boldsymbol{r}_{\boldsymbol{H}}(\boldsymbol{\AA})$ & $\boldsymbol{\tau}_{\boldsymbol{R}}(\boldsymbol{\mu} \mathbf{s})$ & $\boldsymbol{r}_{\boldsymbol{H}}(\boldsymbol{\AA})$ & $\boldsymbol{\tau}_{\boldsymbol{R}}(\boldsymbol{\mu} \mathbf{s})$ & $\boldsymbol{r}_{\boldsymbol{H}}(\AA)$ & $\boldsymbol{\tau}_{\boldsymbol{R}}(\boldsymbol{\mu} \mathbf{s})$ & $\boldsymbol{r}_{\boldsymbol{H}}(\AA)$ & $\boldsymbol{\tau}_{\boldsymbol{R}}(\boldsymbol{\mu} \mathbf{s})$ \\
\hline $\mathbf{1 5}^{\mathbf{0}} \mathbf{C}$ & 37.4 & 8.0 & 35.0 & 13.1 & 35.3 & 11.4 & 60.0 & 11.7 \\
\hline $\mathbf{2 0}^{\mathbf{0}} \mathbf{C}$ & 38.5 & 7.7 & 35.9 & 11.6 & 35.5 & 10.4 & 57.3 & 9.6 \\
\hline
\end{tabular}




\begin{tabular}{|c|c|c|c|c|c|c|c|c|}
\hline $25^{\circ} \mathrm{C}$ & 37.7 & 7.8 & 35 & 10.4 & 34.0 & 9.8 & 61.6 & 9.4 \\
\hline $30{ }^{\circ} \mathrm{C}$ & 38.6 & 7.2 & 34.3 & 9.1 & 35.9 & 8.8 & 58.1 & 8.2 \\
\hline $35^{\circ} \mathrm{C}$ & 39.0 & $\begin{array}{l}6.8 \\
\end{array}$ & 34.4 & 7.54 & 36.1 & 7.7 & 61.0 & 11.9 \\
\hline $40{ }^{\circ} \mathrm{C}$ & 39.3 & 6.4 & 34.9 & 6.8 & 36.2 & 7.1 & 69.0 & 13.8 \\
\hline $45^{\circ} \mathrm{C}$ & 39.6 & 6.5 & 35.5 & 7.2 & 37.1 & 7.4 & 76.0 & 14.8 \\
\hline $50^{\circ} \mathrm{C}$ & 42.6 & 7.5 & 36.1 & 9.1 & 39.1 & 9.0 & 82.0 & 14.7 \\
\hline $55^{\circ} \mathrm{C}$ & 45.5 & 8.5 & 37.6 & 10.9 & 42.0 & 10.5 & 85.7 & 16.2 \\
\hline $60^{\circ} \mathrm{C}$ & 50.4 & 8.9 & 38.1 & 13.8 & 44.0 & 13.2 & 87.3 & 18.3 \\
\hline $65^{\circ} \mathrm{C}$ & 55.4 & 9.3 & 40.5 & 14.9 & 45.4 & 16.0 & 90.9 & 19.2 \\
\hline $70^{\circ} \mathrm{C}$ & 59.1 & 10.4 & 42.6 & 15.3 & 47.0 & 17.9 & 94.1 & 21.1 \\
\hline
\end{tabular}

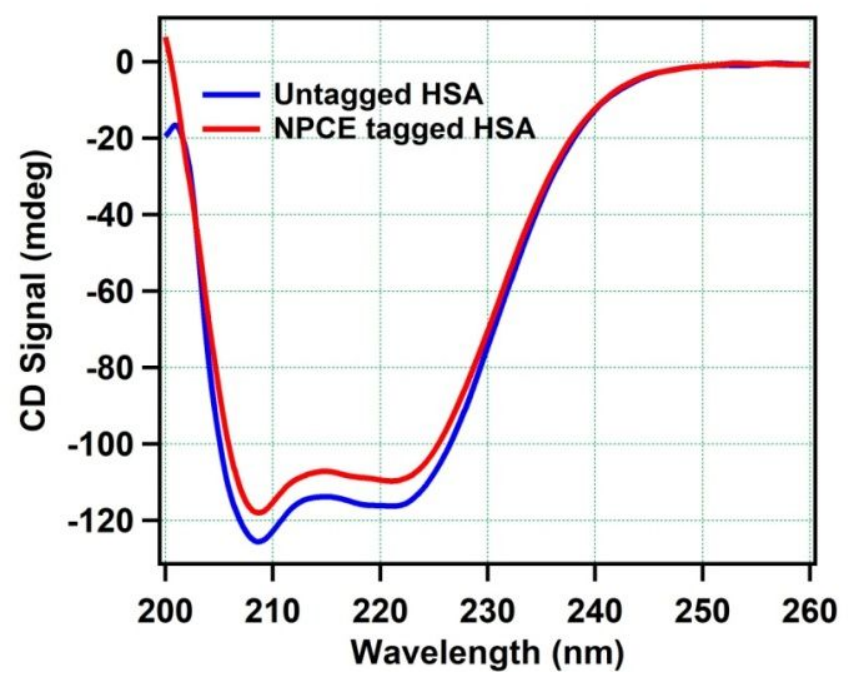

Figure S1: Circular dichroism spectra of untagged (blue) and NPCE tagged (red) HSA. 


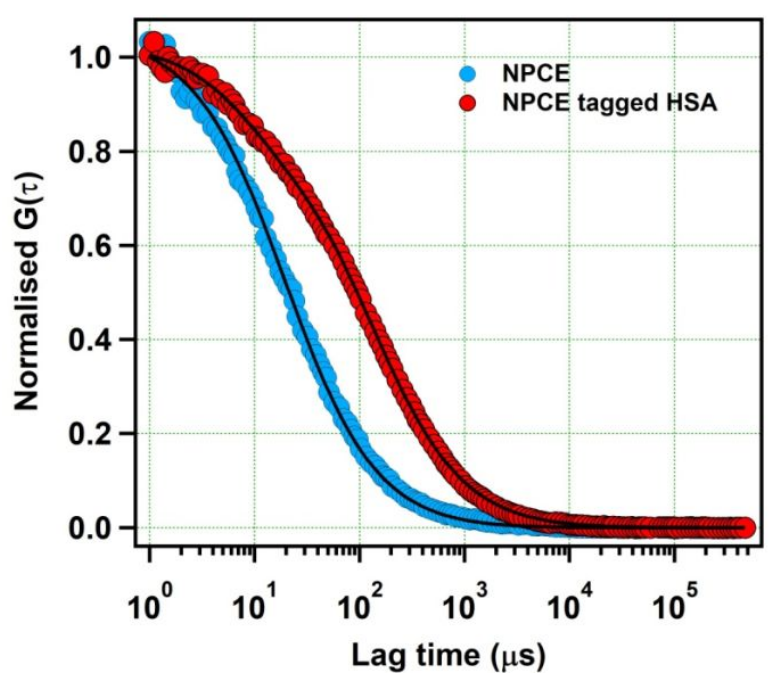

Figure S2: Autocorrelation curve of free NPCE (cyan) and NPCE tagged to HSA (red) in buffer.

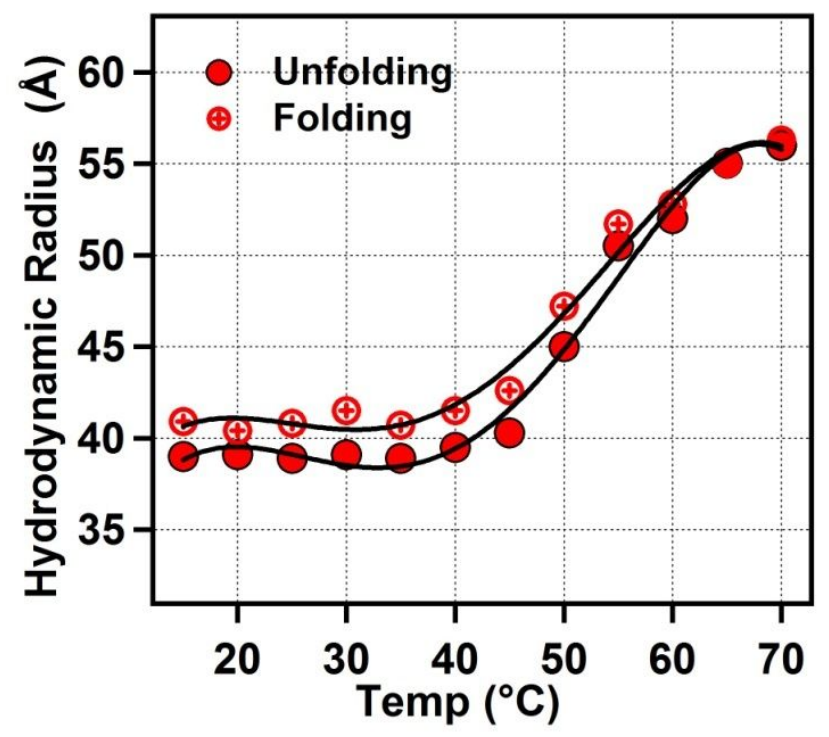

Figure S3: Variation of Hydrodynamic radius of NPCE tagged HSA with increasing the temperature from $15{ }^{\circ} \mathrm{C}$ to $70{ }^{\circ} \mathrm{C}$ (unfolding) and variation of Hydrodynamic radius of NPCE tagged HSA with decreasing temperature from $70{ }^{\circ} \mathrm{C}$ to $15{ }^{\circ} \mathrm{C}$ (Folding). 


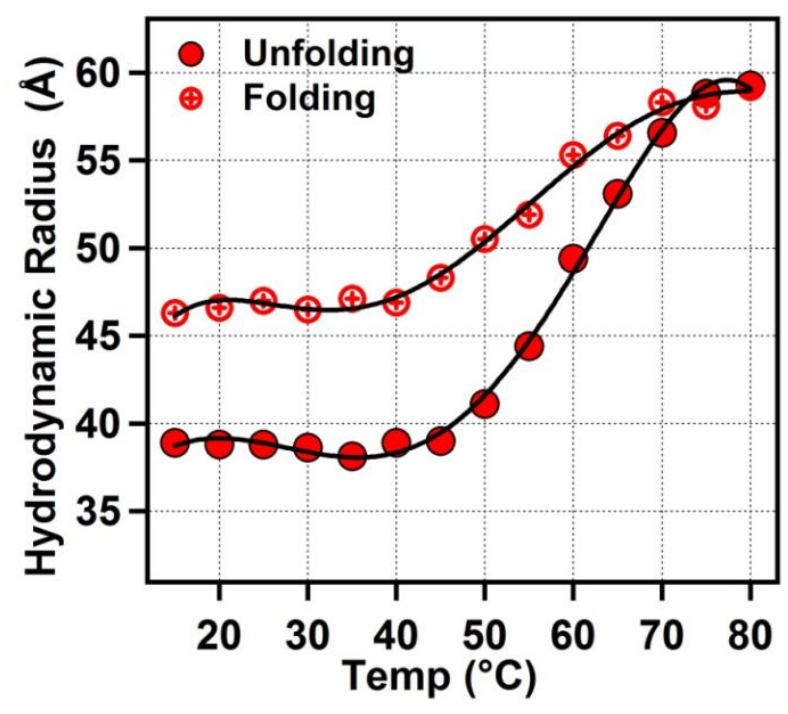

Figure S4: Variation of Hydrodynamic radius of NPCE tagged HSA with increasing the temperature from $15{ }^{\circ} \mathrm{C}$ to $80{ }^{\circ} \mathrm{C}$ (unfolding) and variation of Hydrodynamic radius of NPCE tagged HSA with decreasing temperature from $80{ }^{\circ} \mathrm{C}$ to $15{ }^{\circ} \mathrm{C}$ (Folding).

References:

[R1] N. Hagag, E. R. Birnbaum, D. W. Darnall, Resonance Energy Transfer between Cysteine-34, Tryptophan-214, and Tyrosine-411 of Human Serum Albumin, Biochemistry 22 (1983) 2420-2427. 